\title{
Escala de Avaliação da Motivação para Aprender de Alunos do Ensino Fundamental (EMA)
}

\author{
Scale for Evaluation of Motivation to Learn \\ for Elementary School Students (SML)
}

Edna Rosa Correia Neves* \& Evely Boruchovitch ${ }^{* *}$

Universidade Estadual de Campinas, Campinas, Brasil

\begin{abstract}
Resumo
Este estudo descreve os passos relativos à construção de uma escala para avaliar a motivação para aprender de alunos brasileiros do ensino fundamental e apresenta a análise preliminar de suas propriedades psicométricas. Participaram das duas etapas, 771 estudantes de $2^{\mathrm{a}}$ a $8^{\mathrm{a}}$ séries do ensino fundamental de escolas públicas de Campinas, SP. O alpha de Cronbach foi de 0,80, indicando uma boa consistência interna do instrumento. Pela análise fatorial exploratória, obteve-se uma escala de dois fatores: motivação intrínseca (MI) e motivação extrínseca (ME) que explicaram, respectivamente, 17,68\% e 12,71\% da variância total. Discute-se a necessidade de se aprofundar estudos sobre a viabilidade de utilização da escala para diagnóstico, intervenção e prevenção de problemas motivacionais no contexto escolar.

Palavras-chave: Motivação para aprender; motivação intrínseca; motivação extrínseca; construção de instrumento; ensino fundamental.
\end{abstract}

\begin{abstract}
This study describes the steps of constructing a motivation to learn scale for elementary school students and presents a preliminary analysis of its psychometric properties. It was carried out in two stages, employing 771 students from 2nd to 8 th grade of elementary school level of public schools of Campinas, SP. The alpha of Cronbach was 0,80 indicating a good internal consistency of the scale. The exploratory factorial analysis identified two factors: intrinsic motivation (IM) and extrinsic motivation (EM) explaining respectively $17,68 \%$ and $12,71 \%$ of the total variance. Data is discussed in terms of the need of deepening our knowledge regarding the viability of using the scale for diagnosis, intervention and prevention of motivational problems in the school context.

Keywords: Motivation to learn; intrinsic motivation; extrinsic motivation; construction of instruments; elementary education.
\end{abstract}

Desde o nascimento, o ser humano apresenta interesse e curiosidade, exibindo uma prontidão para aprender e explorar. Esta tendência motivacional é natural, sendo um elemento primordial para seu desenvolvimento cognitivo, social e afetivo. Nos últimos anos, as investigações sobre a motivação para a aprendizagem e os fatores relacionados ao desempenho escolar têm sido revistos por educadores e psicólogos (Bzuneck, 2004). As teorias sociocognitivas da motivação para a aprendizagem têm demonstrado a existência de pelo menos duas formas principais de motivação: a intrínseca e a extrínseca (Amabile, Hill, Hennessey \& Tighe, 1994; Csikszentmihalyi \& Nakamura, 1989; Fortier, Vallerand \& Guay, 1995; Harackiewicks \& Elliot, 1993; Mandelink \& Harackiewicz, 1984). Um aluno é intrinsecamente motivado quando se mantém na tarefa pela

\footnotetext{
"Endereço para correspondência: Rua Paulo Vianna de Souza, no 270, Apto F-11, Campinas, SP, 13060-726. E-mail:ednacneves@yahoo.com.br/E-mail:evely@unicamp.br As autoras agradecem o apoio financeiro da CAPES e do CNPq (proc. No 300529/2004-0), bem como às alunas Tania Maria Serafim, Giselle Rodrigues Vieira Ramos Honório e Rebeca Chiacchio Azevedo Fernandes, do curso de Pedagogia da Faculdade de Educação da Universidade Estadual de Campinas pela ajuda na coleta de dados.
}

atividade em si, por esta ser interessante, envolvente e geradora de satisfação. Por outro lado, pode-se dizer que um aluno é extrinsecamente motivado quando o seu objetivo em realizar uma dada tarefa é o de obter recompensas externas, materiais ou sociais.

As atuais descobertas sobre a motivação intrínseca e as formas auto-reguladoras da motivação extrínseca têm sido muito válidas para se ajudar o aluno a alcançar um desempenho mais eficaz na escola (Deci, Vallerand, Pelletier \& Ryan, 1991). Algumas investigações, com diferentes metodologias, têm revelado resultados importantes para a psicologia escolar e educacional no que diz respeito à mensuração da motivação para aprender dos estudantes. Entre os instrumentos disponíveis destacam-se: a Escala de Motivação Intrínseca, desenvolvida por Harter (1981), o Inventário de Motivação Intrínseca Acadêmica para Crianças (CAIMI), o Inventário de Motivação Intrínseca Acadêmica para Crianças Jovens (Y-CAIMI) de Gottfried (1985, 1986, 1990) e o Inventário de Motivação Intrínseca Acadêmica para alunos do Ensino Médio (CAIMI-HS), de Gottfried (1998, citado por Gottfried, Fleming \& Gottfried, 2001). Tentativas mais recentes de ultrapassar os limites dos instrumentos existentes e avaliar a motiva- 
ção para aprender de forma mais precisa foram feitas por Lepper, Corpus e Iyeangar (2005). É sabido também que a motivação para aprender é considerada uma variável-chave para a auto-regulação da aprendizagem, sendo, portanto, muitas vezes, medida como uma subescala de instrumentos relativos à auto-regulação da aprendizagem (Shil, 2005).

A pesquisa desenvolvida por Harter (1981) com três mil estudantes teve como um dos objetivos desenvolver um instrumento para conhecer a motivação da criança em sala de aula. Para a construção do instrumento, foram definidas cinco dimensões bipolares para caracterizar a motivação intrínseca e a motivação extrínseca, a saber: preferência maior por assuntos desafiadores ou fáceis; preferência por trabalhar de forma independente ou por depender de ajuda externa; a aprendizagem por curiosidade ou pelo desejo de agradar ao professor; o estudar pela própria satisfação ou para agradar ao professor e obter boas avaliações, e o uso de critérios internos ou externos para determinar o sucesso e o fracasso escolar. Os resultados indicaram que a Escala de Motivação Intrínseca de Harter (1981), com a consistência interna variando de 0,68 a 0,84, é uma medida fidedigna e válida para avaliar a motivação intrínseca e extrínseca de crianças.

Gottfried (1985), partindo de uma concepção de motivação intrínseca associada ao gostar, ao prazer, ao interesse e à curiosidade, construiu o Children's Academic Intrinsic Motivation Inventory (CAIMI), um inventário com 122 itens, divididos em subescalas, para medir a motivação intrínseca de crianças para a aprendizagem escolar, de modo geral e em domínios específicos do conhecimento (Gottfried, 1985). Utilizado em três estudos com 567 crianças americanas, esse inventário revelou propriedades psicométricas, no mínimo aceitáveis tanto no seu conjunto, quanto em todas as suas subescalas. Foram obtidos coeficientes alphas que variaram de 0,67 a 0,73 para o Estudo $1(n=141)$, de 0,80 a 0,91 para o Estudo $2(n=260)$ e de 0,83 a 0,93 para o Estudo $3(n=166)$.

Tendo como referência o CAIMI (Gottfried 1985, 1986), Gottfried (1990) desenvolveu o Inventário de Motivação Intrínseca Acadêmica para Crianças Jovens (Y-CAIMI), bem como uma outra versão desse instrumento para alunos do Ensino Médio, o CAIMI-HS (Gottfried, 1998, citado por Gottfried et al., 2001). Esses dois instrumentos, muito semelhantes ao original, porém mais apropriados para as faixas etárias a que se destinam, também apresentaram valores elevados de consistência interna que variaram de 0,82 a 0,95 (Gottfried et al., 2001).

Como a literatura reconhece a complexidade das relações entre motivação intrínseca, extrínseca e o desempenho escolar, bem como a possibilidade de que estudantes sejam simultaneamente intrínseca e extrinsecamente motivados (Harackiewicz, Barron, Pintrich, Elliot \& Thrash, 2002; Wolters, 2004), Lepper et al. (2005) recentemente modificaram a versão original do instrumento de Harter (1981) com o intuito de construir medidas independentes da motivação intrínseca e extrínseca. As duas escalas do tipo Likert desenvolvidas por esses autores foram aplica- das em 797 estudantes de $3^{\text {a }}$ a $8^{\text {a }}$ série de escolas públicas americanas. Apresentaram boas propriedades psicométricas, com a consistência interna variando de 0,78 para a motivação extrínseca a 0,90 para a motivação intrínseca. A escala de motivação intrínseca apontou uma possível estrutura unifatorial desse constructo, visto que os itens relativos ao desafio, à curiosidade e ao trabalho independente carregaram todos no mesmo fator. Já na escala de motivação extrínseca emergiram três fatores: facilidade da tarefa, agradar ao professor e dependência do professor.

Como a maioria dos estudos e instrumentos existentes para avaliar a motivação para aprender de alunos são estrangeiros, o presente artigo descreve dois estudos relativos à construção de uma escala para avaliar a motivação para aprender de alunos brasileiros do Ensino Fundamental. O estudo 1 relata brevemente a construção de um conjunto com 12 pranchas de caráter projetivo para avaliar as orientações motivacionais de alunos do Ensino Fundamental e sua relação com a construção da escala (Neves, 2002; Neves \& Boruchovitch, 2004a, 2004b). O estudo 2 descreve em detalhes a construção da escala e apresenta a análise preliminar de suas propriedades psicométricas.

\section{Estudo 1 - A Construção das Pranchas para Avaliação das Orientações Motivacionais de Crianças e sua Relação com a Construção da Escala}

\section{A Construção do Instrumento: Uma Breve Descrição}

Como descrito em Neves e Boruchovitch (2004b), o instrumento consiste de um conjunto de 12 pranchas com histórias de personagens e ilustrações coloridas com 21,59 $\mathrm{cm}$ de largura e $27,94 \mathrm{~cm}$ de altura, sendo 5 histórias relacionadas à motivação intrínseca, 5 relacionadas à motivação extrínseca e 2 relacionadas à motivação no contexto da progressão continuada. As pranchas foram desenvolvidas e organizadas a partir da literatura da área, tendo como base as principais características da motivação intrínseca e extrínseca (Amabile et al., 1994; Csikszentmihalvi \& Nakamura, 1989; Fortier et al., 1995; Harackiewicks \& Elliot, 1993; Mandelink \& Harackiewicz, 1984).

As principais características da motivação intrínseca levadas em conta para organização das pranchas foram a realização da tarefa associada: à satisfação, ao interesse, ao desafio, à curiosidade e à novidade. A obtenção de recompensas externas, materiais ou sociais, visando a reconhecimento, demonstração de competência ou habilidades em relação a outras pessoas foi o aspecto que serviu de conteúdo para a elaboração das pranchas, no que diz respeito à motivação extrínseca (Neves \& Boruchovitch, 2004b).

Após ouvirem as histórias, os alunos eram solicitados, num primeiro momento, a concordar ou discordar dos personagens da história e, num segundo momento, a justificar suas respostas. As justificativas verbais fornecidas pelos participantes tinham como objetivo transcender a mera análise da concordância ou discordância em relação aos personagens e precisar o tipo predominante de moti- 
vação para aprender dos estudantes, numa tentativa de minimizar respostas socialmente desejáveis (Blumenfeld, Pintrich \& Hamilton, 1986; Harter, 1981). Assim, as perguntas propostas nas pranchas foram as seguintes: Você acha que (o) (a) personagem da história está certo (a)? Sim ou Não? Por que? (Neves \& Boruchovitch, 2004b).

\section{Método}

\section{Participantes}

Fizeram parte do estudo 310 alunos de $2^{\mathrm{a}}(40), 3^{\mathrm{a}}(50)$, $4^{\mathrm{a}}(40), 5^{\mathrm{a}}(5 \mathrm{O}), 6^{\mathrm{a}}(40), 7^{\mathrm{a}}(5 \mathrm{O})$ e $8^{\mathrm{a}}(4 \mathrm{O})$ séries do Ensino Fundamental da rede pública estadual de Campinas, SP. Os participantes eram de ambos os sexos, sendo que 166 $(53,5 \%)$ do gênero feminino e 144 (46,5\%) do masculino. Em relação à faixa etária, 90 (29,0\%) tinham idade entre 13 e 16 anos, seguidos por $95(30,6 \%)$ na faixa de 7 a 9 anos e $125(40,4 \%)$ na faixa de 10 a 12 anos. A faixa etária variou de 7 a 16 anos $(M=11,00, D P=2,34)$. A maioria dos participantes desse grupo não havia repetido nenhuma série escolar $(85,8 \%)$.

\section{Procedimento de Coleta de Dados}

A coleta de dados foi realizada nos anos de 2001 e 2003 em três escolas da cidade de Campinas, SP. A segunda coleta teve como propósito ampliar a amostra e verificar se os dados obtidos no primeiro momento (2001) apresentariam congruência, numa segunda investigação, o que de fato ocorreu (Neves \& Boruchovitch, 2004b). Toda coleta de dados foi precedida por um estudo piloto realizado com 16 alunos (de $2^{\mathrm{a}}$ a $8^{\mathrm{a}}$ série), que não fizeram parte do estudo. Não se detectou a necessidade de revisão do instrumento e confirmou-se a importância de se coletar esse tipo de dado mediante entrevista individual na qual as histórias eram lidas para os alunos, pelo entrevistador.

Antes do início das coletas, obteve-se uma lista de todas as salas de aula das 3 escolas. Em cada escola selecionada, os participantes foram sorteados aleatoriamente de todas as salas de aula das séries em questão. Foi enviado aos pais ou responsáveis pelos estudantes um termo de consentimento esclarecendo a forma de participação. O termo de consentimento continha a descrição das etapas da presente pesquisa e considerações éticas conforme determinações do Conselho Nacional de Saúde (Resolução 196/ 96 e suas complementares).

Após estabelecer um bom rapport com os participantes, o instrumento foi administrado como parte de uma entrevista individual, estruturada, sendo as respostas dos estudantes transcritas na íntegra pelo entrevistador. A coleta de dados teve duração de 30 a 40 minutos, variando em função das séries e idades dos alunos.

\section{Procedimento de Análise de Dados e Resultados}

As respostas dos alunos às pranchas foram estudadas por análise de conteúdo (Bardin, 1977/1991; Berelson, 1952). Foi elaborado um sistema de categorização de respostas para cada história, levando-se em conta as particularidades dos dados e a literatura existente na área (Amabile et al., 1994; Csikszentmihalyi \& Nakamura, 1989; Fortier et al., 1995; Harackiewicks \& Elliot, 1993; Mandelink \& Harackiewicz, 1984). A fim de maximizar a consistência da análise de dados, cada categoria foi definida operacionalmente, além de ter sido elaborado pelas autoras, um conjunto de regras para classificação de cada resposta nas categorias existentes (Neves, 2002).

Como resultado da análise de conteúdo, surgiram 14 categorias de respostas: (1) a escola deve reprovar o aluno, (2) a importância da novidade para a aprendizagem, (3) assuntos fáceis como um valor importante, (4) esforço como um valor importante, (5) estudo como desnecessário, (6) estudo como meio de evitar repetência futura, (7) estudo como um valor importante, (8) estudo como uma obrigação, (9) estudo por medo da opinião externa, (10) evitar fracassos futuros como um valor importante, (11) não é importante ser o melhor aluno da sala de aula, (12) não ser curioso como um valor importante, (13) nota como um valor importante, (14) recompensas como um valor importante. Para cada prancha ocorreram em média 3 categorias de respostas. As categorias estudo como um valor importante, esforço como um valor importante e estudo como meio de evitar repetência futura foram comuns em várias pranchas.

A consistência do processo de categorização foi avaliada mediante utilização de três juízes independentes que analisaram 96 respostas selecionadas aleatoriamente para serem representativas dos dois tipos de orientações motivacionais, cabendo a avaliação de 34 respostas para cada juiz. A porcentagem de correspondência atingida entre o pesquisador e os juízes nas 96 respostas foi de 93\% (Neves \& Boruchovitch, 2004b).

Um estudo acerca da consistência interna das respostas dos participantes em relação às 12 pranchas foi realizado. Procurou-se verificar se o participante que apresentava uma orientação motivacional extrínseca numa prancha relacionada à motivação extrínseca continuava com essa orientação em outras relacionadas à motivação extrínseca. O mesmo procedimento de análise foi realizado no que concerne às pranchas cujos conteúdos eram relativos à motivação intrínseca. A análise das respostas dos participantes revelou que a maioria dos participantes $(87,8 \%)$ foi muito consistente nas suas respostas no instrumento inteiro (Neves \& Boruchovitch, 2004b).

Como a maioria dos instrumentos para mensurar as orientações motivacionais de estudantes disponíveis na literatura são estrangeiros, as pranchas baseadas em questões abertas foram úteis para mapear as orientações motivacionais de alunos brasileiros do Ensino Fundamental. Assim, as categorias estudo como um valor importante, esforço como um valor importante e estudo como meio de evitar repetência futura, dentre outras que emergiram em várias pranchas do estudo 1 serviram de base para a redação de itens para um outro instrumento: A Escala de Avaliação da Motivação para Aprender de Alunos do Ensino Fundamental (EMA). Na realidade, o uso de questões abertas como uma etapa anterior à construção de 
instrumentos com itens fechados vem sendo bastante recomendado pela literatura (Boruchovitch \& Schall, 1999; Boruchovitch et al., 2006; Günther \& Lopes Jr., 1990; Isaac \& Michael, 1982).

Diferentemente das pranchas, a EMA permite, por sua vez, uma coleta de dados mais rápida, mais objetiva e autoreferenciada, uma vez que, nas pranchas, os alunos não avaliaram a própria motivação e sim a de personagens fictícios com os quais, possivelmente, se identificavam ou não. Assim sendo, baseando-se nas informações obtidas em estudos anteriores com questões abertas e na literatura da área, a primeira versão da EMA foi construída por Neves e Boruchovitch, em 2004 (Neves \& Boruchovitch, 2005; Neves, Boruchovitch, Serafim \& Honório, 2005).

\section{Estudo 2 - A Construção da Escala de Avaliação da Motivação para Aprender de Alunos do Ensino Fundamental (EMA)}

Descrição dos Principais Passos para a Construção da Escala

Inicialmente, foram redigidos 36 itens cujas respostas envolviam de múltipla escolha, tendo como alternativas: sempre, às vezes e nunca. Os itens foram confeccionados na primeira pessoa tendo como objetivo avaliar a orientação geral para aprendizagem escolar de alunos do Ensino Fundamental. Após a redação dos itens, o conteúdo de cada um deles foi avaliado e classificado como intrínseco, extrínseco ou não se aplica por 4 juízes independentes. A avaliação dos juízes contribuiu para a reformulação de alguns itens que apresentavam redação ambígua e exclusão de 2 itens classificados pelos mesmos como não se aplica. A porcentagem de correspondência atingida entre os juízes variou de $85 \%$ a $100 \%$.

A escala foi pré-testada em 12 alunos de uma escola pública da cidade de Campinas, SP. O estudo piloto detectou a necessidade de revisão do instrumento no que diz respeito a pequenos problemas de linguagem. Após a avaliação dos juízes e a conclusão do estudo piloto, desenvolveu-se a primeira versão da escala com 34 itens fechados. Os 34 itens foram propostos em forma de escala do tipo Likert. As questões ímpares tinham como objetivo avaliar a motivação intrínseca e as pares, a motivação extrínseca. Um exemplo de uma questão da escala é: Eu estudo porque estudar é importante para mim: ( ) sempre ( ) às vezes ( ) nunca. A alternativa, «sempre» vale 3 pontos para as questões referentes às orientações motivacionais intrínsecas e 1 ponto para as extrínsecas. A alternativa «nunca» vale 1 ponto para as questões relativas às orientações motivacionais intrínsecas e 3 para as extrínsecas e a alternativa "às vezes" vale 2 pontos para todas as questões. O valor máximo de pontos a ser obtido na escala é de 102 e o valor mínimo é de 34. Quanto maior é a pontuação do estudante na escala, maior é a sua orientação motivacional intrínseca. A escala é acompanhada também de um conjunto de instruções simples de forma a se padronizar sua aplicação.

\section{Método}

\section{Participantes}

Participaram do presente estudo 461 alunos de $2^{\text {a }}(50)$, $3^{\mathrm{a}}(57), 4^{\mathrm{a}}(50), 5^{\mathrm{a}}(126), 6^{\mathrm{a}}(114), 7^{\mathrm{a}}(32)$ e $8^{\mathrm{a}}(32)$ séries do Ensino Fundamental da rede pública estadual de Campinas, SP. Os participantes eram de ambos os sexos, sendo que $264(57,3 \%)$ do gênero feminino e 197 (42,7\%) do masculino. Em relação à faixa etária, 252 (54,7\%) tinham idade entre 11 e 13 anos, seguidos por 157 (34,1\%) na faixa de 7 a 10 anos e $52(11,3 \%)$ na faixa de 14 a 17 anos. A faixa etária variou de 7 a 17 anos $(M=11,00, D P=1,83)$. A maioria dos participantes desse grupo não havia repetido nenhuma série escolar (91,3\%).

\section{Procedimento de Coleta de Dados}

A coleta de dados foi realizada nos anos de 2004 e 2005 em cinco escolas públicas da rede estadual e municipal da cidade de Campinas, SP, por aplicadores especialmente treinados para a tarefa. Foi enviado aos pais ou responsáveis pelos alunos das séries estudadas um termo de consentimento, esclarecendo os objetivos da pesquisa e a forma de participação, encaminhado por intermédio do próprio aluno. Foram convocados para a coleta de dados apenas os estudantes cujos responsáveis autorizaram a participação. Assim como no estudo 1, foram seguidos os cuidados éticos recomendados pelo Conselho Nacional de Saúde (Resolução 196/96 e suas complementares).

Os dados foram coletados coletivamente. As instruções e as questões da escala eram lidas pelos aplicadores e ouvidas pelos participantes. Os alunos eram orientados sobre o preenchimento da escala e para que levantassem a mão, caso tivessem dúvidas. Foi recomendado o uso de régua para facilitar a leitura e melhor acompanhar o preenchimento das respostas na escala. A coleta de dados teve duração de 15 a 25 minutos, variando em função das séries e idades dos alunos.

\section{Resultados e Discussão}

Inicialmente, para se examinar a consistência interna da EMA, o alpha de Cronbach foi calculado para amostra total ( $N=461)$, tendo-se obtido um alpha de 0,82 , indicando que essa escala apresenta um bom índice de consistência interna (Prieto \& Muñiz, 2000).

Para avaliar a estabilidade temporal da EMA foi feita uma reaplicação da mesma, após 15 dias da primeira aplicação, em 49 alunos, selecionados aleatoriamente, sendo 7 por série. Resultados semelhantes foram alcançados no que concerne à estabilidade temporal da escala, em medidas de teste e reteste (alpha de Cronbach = 0,80). Foi também calculada a correlação de Pearson entre as duas aplicações, tendo-se encontrado uma correlação elevada (Hinkle, Wiersma \& Jurs, 1998) e altamente significativa entre as duas aplicações $(\mathrm{r}=0,71 ; p=0,00)$. Em linhas gerais, tanto a consistência interna da EMA, quanto a sua estabilidade temporal assemelham-se aos valores obtidos nas escalas 
para crianças das pesquisas estrangeiras (Gottfried, 1985, 1986, 1990; Gottfried et al., 2001; Harter, 1981; Lepper et al., 2005; Shil, 2005).

Em seguida, os estudos para a validação da escala foram realizados por meio do programa Statistical Package for the Social Sciences (SPSS), versão 10.0. Para verificar a fatorabilidade da escala, efetuou-se o Teste de Esfericidade de Bartlett e o Teste de Kaiser-Meyer-Olkin (KMO).

O Teste de Esfericidade de Bartlett indicou uma correlação entre os itens $\left(\mathrm{X}^{2}[561, N=461]=4061,609\right.$; $p<0,000)$. A medida de adequação da amostra, averiguada pelo índice de Kaiser-Meyer-Olkin (KMO), foi de 0,854. Os dados obtidos por esses dois procedimentos revelaram que há um nível de probabilidade muito adequado para a correlação entre variáveis e que a amostra é apropriada para a realização da Análise Fatorial (Pereira, 2001).

Utilizou-se a análise fatorial exploratória para levantar a estrutura fatorial da medida. Empregou-se o método dos componentes principais (eigenvalue superior a 2,0). Aplicou-se, a rotação Varimax. Para obter a matriz rotada foi estipulado o critério de carga fatorial mínima de 0,30 para a inclusão dos itens (Crocker \& Algina, 1986; Kline, 1994). Para avaliar a conveniência da manutenção do número de fatores estipulados, utilizou-se o Scree Test.

Como resultado da análise dos componentes principais (PC), obteve-se uma escala de dois fatores. A estrutura mais adequada foi a bifatorial. Foi possível denominá-los como: Fator 1 - Motivação Intrínseca e Fator 2 - Motivação Extrínseca. O Fator 1, Motivação Intrínseca, foi composto pelos 17 itens ímpares, originalmente propostos (1, $3,5,7,9,11,13,15,17,19,21,23,25,27,29,31$ e 33) com saturação variando entre 0,42 e 0,68. Apresentou eigenvalues de 6,00, explicando $17,68 \%$ da variância total. Já o Fator 2, Motivação Extrínseca, foi composto por 14 itens, dos 17 iniciais $(2,4,8,10,12,14,16,18,20,24,26$, 28, 30 e 32) com saturação variando entre 0,33 e 0,71. Apresentou eigenvalues de 4,32, explicando 12,71\% da variância total. Os dois fatores identificados permitiram que apenas 30,39\% da variabilidade da escala fosse explicada. Por ser um percentual abaixo do desejável (< $40 \%$ ), sugere-se que novos estudos sejam feitos, visando ao melhor esclarecimento sobre a mensuração do construto em apreço.

Por razões distintas, foram excluídos 3 itens: o 6, o 22 e o 34. O item 6 (eu estudo para ter um bom emprego no futuro) não alcançou a carga fatorial mínima recomendada pela literatura (Crocker \& Algina, 1986; Kline, 1994). Os itens 22 (eu prefiro estudar assuntos fáceis) e 34 (eu desisto de fazer uma tarefa quando encontro dificuldade), ambos carregaram nos dois fatores, com carga fatorial maior no Fator 1 e não no 2, como seria inicialmente esperado, contrastando-se com os resultados encontrados na literatura da área (Harter, 1981; Lepper et al., 2005). Mas, como argumentam Lepper et al. (2005), uma vez que as relações entre a motivação extrínseca e a intrínseca são bem complexas, é possível que coexistam o prazer, o desafio, a busca de tarefas fáceis (nas quais o sucesso é assegurado) e a tendência a desistir diante de impasses.

Assim, a escala construída inicialmente com 34 itens foi substituída por uma com 31 itens após a análise dos componentes principais. Os principais resultados dessa análise podem ser vistos na Tabela 1.

Tabela 1

Distribuição dos Itens por Fator com a Carga Fatorial Apresentada

\begin{tabular}{ll}
\hline Itens da Escala & Cargas Fatoriais \\
\cline { 2 - 2 } & Fator 1 \\
\hline 1. Eutor 2 \\
\hline 3. Eu tenho vontade de conhecer e aprender assuntos novos & 0,448 \\
\hline 5. Eu gosto de estudar assuntos desafiantes & 0,451 \\
\hline 7. Eu gosto de estudar assuntos difíceis & 0,421 \\
\hline 9. Eu me esforço bastante nos trabalhos de casa, mesmo sabendo que não vão valer como nota & 0,588 \\
\hline 11. Eu estudo mesmo sem os meus pais pedirem & 0,619 \\
\hline 13. Eu me esforço bastante nos trabalhos, em sala de aula, mesmo sabendo que não vai valer como nota & 0,499 \\
\hline 15. Eu estudo porque estudar me dá prazer e alegria & 0,673 \\
\hline 17. Eu fico tentando resolver uma tarefa, mesmo quando ela é difícil para mim & 0,537 \\
\hline 19. Eu prefiro aprender, na escola, assuntos que aumentem minhas habilidades ou meus conhecimentos & 0,527 \\
\hline 21. Eu faço minhas lições de casa, mesmo que meus pais não me peçam & 0,487 \\
\hline 23. Eu estudo porque gosto de ganhar novos conhecimentos & 0,618 \\
\hline 25. Eu gosto de estudar & 0,687 \\
\hline 27. Eu procuro saber mais sobre os assuntos que gosto, mesmo sem minha professora pedir & 0,530 \\
\hline 29. Eu gosto de ir para a escola porque aprendo assuntos interessantes lá & 0,588 \\
\hline
\end{tabular}




\begin{tabular}{ll}
\hline 31. Eu estudo porque quero aprender cada vez mais & 0,603 \\
\hline 33. Eu fico interessado (a) quando a professora começa uma lição nova & 0,598 \\
\hline 2. Eu estudo por medo dos meus pais brigarem comigo & 0,335 \\
\hline 4. Eu faço os deveres de casa por obrigação & 0,447 \\
\hline 8. Eu estudo porque meus pais prometem me dar presentes, se as minhas notas forem boas & 0,599 \\
\hline 10. Eu estudo porque minha professora acha importante & 0,625 \\
\hline 12. Eu estudo porque fico preocupado(a) que as pessoas não me achem inteligente & 0,595 \\
\hline 14. Eu estudo por medo dos meus pais me colocarem de castigo & 0,530 \\
\hline 16. Eu só estudo para não me sair mal na escola & 0,434 \\
\hline 18. Eu estudo para os meus pais deixarem eu ir brincar com os meus amigos & 0,657 \\
\hline ou fazer as coisas que eu gosto & 0,590 \\
\hline 20. Eu só estudo para agradar meus professores & 0,338 \\
\hline 24. Eu estudo apenas aquilo que a professora avisa que vai cair na prova & 0,711 \\
\hline 26. Eu só faço meus deveres de casa porque meus pais acham importante & 0,341 \\
\hline 28. Eu só estudo porque quero tirar notas altas & 0,606 \\
\hline 30. Eu só estudo porque meus pais mandam & 0,522 \\
\hline 32. Eu estudo por obrigação & \\
\hline
\end{tabular}

Nota. Resultados obtidos através da análise de dados com o SPSS.

O número de fatores identificados mediante a análise do Scree Test coincidiu com o número de fatores fixados. Assim sendo, decidiu-se pela manutenção dos dois fatores, considerando principalmente a coerência teórica dos itens e o gráfico de sedimentação obtido na análise realizada.

Com relação à precisão, a escala total alcançou um índice satisfatório, com coeficiente de Cronbach igual a 0,80. $O$ primeiro fator alcançou o índice de $\alpha=0,86$ e o segundo fator de $\alpha=0,80$. Os 31 itens finais, ao se agruparem em dois fatores, revelam congruência da escala com os constructos teóricos que ela se propõe a medir.

Parece pertinente considerar para este conjunto de itens uma estrutura bifatorial: Fator 1 - Motivação Intrínseca (MI) e Fator 2 - Motivação Extrínseca (ME). Os 17 itens contidos no Fator 1 apresentam, de modo geral, as principais características da motivação intrínseca e os 14 do Fator 2, as principais características da motivação extrínseca (Amabile et al., 1994; Csikszentmihalyi \& Nakamura, 1989; Fortier et al., 1995; Harackiewicks \& Elliot, 1993; Harter, 1981; Lepper et al., 2005; Mandelink \& Harackiewicz, 1984). Estimou-se, também, no presente estudo, a correlação entre os Fatores 1 e 2, mediante o cálculo do coeficiente de Pearson, tendo-se encontrado uma correlação fraca e não significativa entre eles $(\mathrm{r}=0,24 ; p=0,60)$. Na EMA, esses fatores emergiram bem definidamente e com uma relativa independência entre si. A EMA parece ter se assemelhado mais, em sua composição de itens, às escalas de Lepper et al. (2005), nas quais os alunos se auto-avaliavam em relação a ambos os comportamentos intrínseca e extrinsecamente motivados, não tendo que necessariamente escolher qual desses comportamentos melhor os representa, como ocorreu no instrumento de Harter (1981).

A construção da presente escala sustentou-se nas teorias sociocognitivas da motivação para a aprendizagem que têm demonstrado a existência de pelo menos dois tipos principais de motivação para aprender, a intrínseca e a extrínseca (Amabile et al., 1994; Csikszentmihalyi \& Nakamura, 1989; Fortier et al., 1995; Harackiewicks \& Elliot, 1993; Mandelink \& Harackiewicz, 1984). Foram esses dois tipos de motivação que se buscou medir e que foram encontrados na escala para alunos do Ensino Fundamental descrita neste estudo. Entretanto, ao realizar uma revisão da literatura especializada, Guimarães (2004) aponta que estudos baseados na Teoria da Autodeterminação têm apresentado novas considerações sobre a motivação extrínseca e mais especificamente sobre as suas formas auto-reguladoras como: regulação externa, introjetada, identificada e integrada. Tem-se constatado que o comportamento extrinsecamente motivado também pode ser autodeterminado, não sendo necessariamente sempre negativo para a aprendizagem (Deci \& Ryan, 1995; Rigby, Deci, Patrick \& Ryan, 1992; Ryan \& Deci, 2000; Ryan \& Stiller, 1991).

Como as formas auto-reguladoras da motivação extrínseca não foram avaliadas no âmbito do presente estudo, recomenda-se que futuros estudos sejam voltados para a construção de instrumentos ou de novos itens que envolvam a avaliação da validade de constructo dessas formas auto-reguladoras da motivação extrínseca, nas diversas faixas etárias. Avanços nesse sentido, certamente, contribuirão para o aumento do conhecimento acerca da motivação para aprender de alunos brasileiros.

Lepper et al. (2005) também sugerem que pesquisas examinem melhor a motivação intrínseca e a motivação extrínseca para se aquilatar, se são pólos diferentes de um mesmo constructo ou se são constructos diferentes, independentes e/ou complementares. Defendem a construção de medidas nas quais os estudantes indiquem o 
quanto cada comportamento (seja ele intrínseco ou extrínseco) contribuiu para se alcançar um determinado resultado. Um outro ponto levantado por Lepper et al. (2005) é a necessidade de se olhar a motivação intrínseca com mais cuidado, pois ela pode ter dimensões outras além da busca por vontade ou escolha própria. Segundo esses autores, pode haver um conjunto maior de motivos intrínsecos ainda não contemplados nos instrumentos atuais.

Futuras investigações devem também orientar seus esforços para a construção de versões da Escala de Avaliação da Motivação para Aprender de Alunos do Ensino Fundamental voltadas para as áreas específicas do conhecimento, já que é também sabido que a motivação para aprender varia em função dos diferentes conteúdos, podendo um estudante ser altamente motivado em uma disciplina e desmotivado em outra. Acresce-se que os interesses tendem também a se definir melhor com o avançar da escolaridade (Boruchovitch, 2004; Gottfried, 1990; Gottfried et al., 2001).

Se a motivação intrínseca declina com o avançar da escolaridade e se a motivação extrínseca pode também proporcionar aos alunos um bom desempenho na escola são questões que têm sido alvo de inúmeras controvérsias na literatura internacional (Gottfried, 1990; Gottfried et al., 2001; Harackiewicz et al., 2002; Harter, 1981; Lepper et al., 2005; Wolters, 2004). Acredita-se que a EMA poderá ser útil e ter importantes implicações educacionais, se empregada em investigações nacionais que precisem melhor as relações entre os diferentes tipos de motivação e as variáveis-chave como a idade e o desempenho escolar.

Como uma outra direção para pesquisas futuras, considera-se que seria interessante correlacionar os resultados obtidos na presente escala com os dados das Pranchas para Avaliação das Orientações Motivacionais de Crianças. A correlação teria por objetivo verificar se é muito diferente avaliar a própria motivação e a de personagens fictícios.

Conhecer a motivação para aprender e suas relações com medidas de desempenho, estratégias de aprendizagem, auto-eficácia e ansiedade dos alunos, entre outras variáveis, são também exemplos de investigações que poderiam ser conduzidas utilizando-se a EMA.

Certamente, esses estudos correlacionais seriam úteis, não só para se conhecer melhor validade preditiva da EMA, mas também para elucidar as relações existentes entre a motivação para aprender e esses constructos tão relevantes para o processo ensino-aprendizagem.

\section{Considerações Finais}

Espera-se que a escala de avaliação da motivação para aprender desenvolvida no presente estudo represente um passo inicial no que concerne à construção de um instrumento nacional útil para avaliar a orientação geral para aprendizagem escolar de alunos brasileiros do Ensino Fundamental e que possa contribuir para o aumento do conhecimento no que diz respeito à motivação para aprender de estudantes, em nosso meio. Todavia, destaca-se ainda a necessidade de que futuras investigações sejam conduzidas, aplicando a escala em amostras maiores e mais representativas nacionalmente, bem como de que pesquisas sejam voltadas para o exame desse instrumento à luz de novas análises fatoriais exploratórias e confirmatórias. Não há dúvida de que medidas que consigam captar mais sensivelmente a complexidade e a coexistência das diferentes formas de motivação para aprender são necessárias e desejáveis, sobretudo para estudantes no início da escolarização formal. A ampliação de estudos empregando a EMA será promissora no que se refere às possibilidades de identificação de suas potencialidades para diagnóstico, intervenção e prevenção no contexto escolar.

\section{Referências}

Amabile, T. M., Hill, K. G., Hennessey, B. A., \& Tighe E. M. (1994). The work preference inventory: Assessing intrinsic and extrinsic motivation orientation. Journal of Personality and Social Psychology, 6(5), 950-967.

Bardin, L. (1991). Análise de conteúdo (L. A. Reto \& A. Pinheiro, Trad.). São Paulo, SP: Edições 70. (Original publicado em 1977)

Berelson, B. (1952). Content analysis in communications research. Glencoe, IL: Free Press.

Blumenfeld, P. C, Pintrinch, P. R., \& Hamilton, V. L. (1986). Children's concepts of ability, effort and conduct. American Educational Research Journal, 28(10), 95-104.

Boruchovitch, E. (2004). A study of causal attributions for success and failure in mathematics among brazilian students. Revista Interamericana de Psicologia, 38(2), 53-60.

Boruchovitch, E., Santos, A. A. A., Costa, E. R., Neves, E. R. C., Cruvinel, M., Primi, R., \& Guimarães, S. E. R. (2006). Estudo preliminar para construção de uma escala de estratégias de aprendizagem para alunos do ensino fundamental. Revista Psicologia: Teoria e Pesquisa, 22(3), 297-304.

Boruchovitch, E., \& Schall, V. T. (1999). Questionnaires in health education research. Advantages and disadvantages of openended questions. Implications for health research methodology. Ciência e Cultura, 28(1), 12-15.

Bzuneck, J. A. (2004). A motivação do aluno: Aspectos introdutórios. In E. Boruchovitch \& J. A. Bzuneck (Eds.), Motivação do aluno: Contribuições da psicologia contemporânea (3. ed., pp. 09-36). Petrópolis, RJ: Vozes.

Crocker, L., \& Algina, J. (1986). Introduction to classical \& Modern test theory. Orlando, FL: Holt, Rinehart and Winston.

Csikszentmihalyi, M., \& Nakamura, J. (1989). The dynamics of intrinsic motivation: A study of adolescents. In C. Ames \& R. Ames (Eds.), Research on motivation in education: Vol. 3. Goals and cognitions (pp. 249-277). New York: Academic Press.

Deci, E. L., \& Ryan, R. M. (1995). Intrinsic motivation and selfdetermination in human behavior. New York: Plenum.

Deci, E. L., Vallerand, R. J., Pelletier, L. G., \& Ryan, R. M. (1991). Motivation in education: The self-determination perspective. Educational Psychologist, 26(3/4), 325-346.

Fortier, M. S., Vallerand, R. J., \& Guay, F. (1995). Academic motivational and school performance: Toward a structural model. Contemporary Educational Psychology, 20(3), 257-274.

Gottfried, A. E. (1985). Academic intrinsic motivation in elementary and junior high school students. Journal of Educational Psychology, 77(6), 631-645. 
Gottfried, A. E. (1986). Children's Academic Intrinsic Motivation Inventory. Odessa, FL: Psychological Assessment Resources.

Gottfried, A. E. (1990). Academic intrinsic motivation in young elementary school children. Journal of Educational Psychology, 88(3), 525-538.

Gottfried, A. E., Fleming, J. M., \& Gottfried, A. W. (2001). Continuity of academic intrinsic motivation from childhood through late adolescence: A longitudinal study. Journal of Educational Psychology, 93(1), 3-13.

Guimarães, S. E. R. (2004). Motivação intrínseca, extrínseca e o uso de recompensas em sala de aula. In E. Boruchovitch \& J. A. Bzuneck (Eds.), Motivação do aluno: Contribuições da psicologia contemporânea (3. ed., pp. 37-57). Petrópolis, RJ: Vozes.

Guinther, H., \& Lopes, J., Jr. (1990). Perguntas abertas versus perguntas fechadas: Uma comparação empírica. Psicologia: Teoria e Pesquisa, 6(2), 203-213.

Harackiewicz, J. M., Barron, K. E., Pintrich, P. R., Elliot, A. J., \& Thrash, T. M. (2002). Revision of achievement goal theory: Necessary and illuminating. Journal of Educational Psychology, 94(3), 638-645.

Harackiewicks, J. M., \& Elliot, A. J. (1993). Achievement goals and intrinsic motivation. Journal of Personality and Social Psychology, 65(5), 904-915.

Harter, S. (1981). A new self-report scale of intrinsic versus extrinsic orientation in the classroom: Motivational and informational components. Developmental Psychology, 17(3), 300-3 12

Hinkle, D. E., Wiersma, W., \& Jurs, S. G. (1998). Applied statistics for behavioral science. Boston: Houghton Mifflin.

Isaac, S., \& Michael, W. B. (1982). Handbook in research and evaluation. San Diego, CA: Edits I \& M.

Kline, P. (1994). An easy guide to factor analysis. New York: Routledge.

Kuder, G. F., \& Richardson, M. W. (1937). The theory of the estimation of test reliability. Psychometrika, 2(1), 151-160.

Lepper, M. R., Corpus, J. H., \& Iyengar, S. (2005). Intrinsic and extrinsic motivation in the classroom: Age differences and academic correlates. Journal of Educational Psychology, 97(2), 184-196.

Mandelink, G., \& Harackiewicz, J. (1984). Proximal versus distal goal setting and intrinsic motivation. Journal of Personality and Social Psychology, 47(4), 918-928.

Neves, E. R. C. (2002). As orientações motivacionais e as crenças sobre inteligência, esforço e sorte de alunos do ensino fundamental. Dissertação de Mestrado não-publicada, Universidade Estadual de Campinas, SP.

Neves, E. R. C., \& Boruchovitch, E. (2004a). A motivação de alunos no contexto da progressão continuada. Revista Psicologia: Teoria e Pesquisa, 20(1), 77-85.

Neves, E. R. C., \& Boruchovitch, E. (2004b). Construção e análise de um instrumento para avaliar as orientações motivacionais de estudantes brasileiros. In Avaliação Psicológica: Formas e Contextos (Ed.), Trabalhos completos de comunicações científicas, $X$ Conferência Internacional de Avaliação Psicológica: Formas e Contextos (pp. 79-86). Braga, SP: Psiquilíbrios.

Neves, E. R. C., \& Boruchovitch, E. (2005). A motivação para aprender de alunos do ensino fundamental: Uma análise por idade e série escolar [Resumo]. In VII Congresso de Psicologia Escolar e Educacional (Ed.), Resumos de comunicações científicas (Anais Eletrônico). Curitiba, PR.

Neves, E. R. C., Boruchovitch, E. Serafim, T. M., \& Honório, G. R. V. R. (2005). A construção da escala de avaliação da motivação aprender: Um estudo preliminar [Resumo]. In II Congresso de Avaliação Psicológica (Ed.), Resumos de comunicações científicas: Desafios para a formação, prática e pesquisa (Anais Eletrônico). Gramado, RS.
Pereira, J. C. R. (2001). Análise de dados qualitativos: Estratégias metodológicas para as Ciências da Saúde, Humanas e Sociais. São Paulo, SP: EDUSP.

Prieto, G., \& Muñiz, J. (2000). Um modelo para evaluar la calidad de los tests utilizados em España. Retirado em 14 fev. 2006, de http://www.cop.es/tests/modelo.htm

Rigby, C. S., Deci, E. L., Patrick, B. C., \& Ryan, R. M. (1992). Beyond the intrinsic-extrinsic dichotomy: Self-determination in motivation and learning. Motivation and Emotion, 16 (3), 165-185

Ryan, R. M., \& Deci, E. L. (2000). Intrinsic and extrinsic motivations: Classic definitions and new directions. Contemporary Educational Psychology, 25(1), 54-67.

Ryan, R. M., \& Stiller, J. (1991). The social contexts of internalization: Parent and teacher influences on autonomy, motivation, and learning. In C. Ames \& R. Ames (Eds.), Advances in motivation and achievement (Vol. 7, pp. 115-149). Hartford, CT: Jai Press.

Shil, S. S. (2005). The role of achievement goals in children's learning in Taiwan. The Journal of Educational Research, 1(5), 310-319.

Wolters, C. A. (2004). Advancing achievement goal theory: Using goal structures and goal orientations to predict students' motivation, cognition, and achievement. Journal of Educational Psychology, 96(2), 236-250.
Recebido: 13/03/2006

$1^{a}$ revisão: $20 / 10 / 2006$ $2^{a}$ revisão: $21 / 12 / 2006$ Aceite final: 27/12/2006 